\title{
LE TAUX BUTYREUX SANGUIN DES REPRODUCTEURS BOVINS ET SES RAPPORTS ÉVENTUELS AVEC LA VALEUR D'ÉLEVAGE
}

André-M. LEROY,

Ingénieur agronome,

Chef de travaux à l'Institut national agronomique de Paris. par

\author{
J. MARCQ, \\ Doeteur en médecine vétérinaire, \\ Professeur à l'Institut agronomique \\ de Gembloux.
}

avee la collaboration de M. VELINI,

Ingénieur agronome,

Préparateur de la Station volante d'expérimentation zootechnique de l'Office régional du Nord.

et de MM. VALISSANT et G. BARJOT, Ingénieurs agronomes,

Assistants du Laboratoire de zootechnie de l'Institut national agronomique.

Ce travail est un rapport présenté au Congrés de Zootechnie qui s'est tenu à Liège en 1930. A Liège, on a procédé à une revision de nos conceptions modernes en matière d'hérédité dans ce qu'elles pouvaient avoir de plus immédiatement utile pour l'élevage.

Ce rapport vise plus particulièrement les corrélations éventuelles entre la teneur du sang en matières grasses et la valeur pour l'élevage des jeunes reproducteurs mâles et femelles.

Si le patrimoine héréditaire n'est pas en état d'incessante transformation, mais présente au contraire une fixité considérable, on comprendra que la notion de facteurs ou "gènes ", de ces "quelque chose » qui confèrent au gamète une capacité mendélisante s'identifie avec la notion de la constitution cellulaire, et finalement avec la constitution de l'individu, lequel dérive d'une cellule unique, l'œuf. Malheureusement, nous sommes actuellement incapables d'analyser directement le patrimoine héréditaire localisé dans la eellule, et nous employons comme réactif l'étude des ressemblances morphologiques et physiologiques entre plusieurs générations. Si nous pouvions intervenir directement, nous aurions fait un grand pas dans la voie de l'amélioration de nos grandes espèces animales domestiques, en admettant, ce qui est logique, que les rendements divers sont liés à la constitution, c'est-à-dire à la caractéristique anatomo-physiologique de l'organisme, laquelle conditionne l'amplitude et le sens des réactions aux influences extérieures.

Dans la pratique, nous jugeons ordinairement de la valeur d'un animal par les moyens suivants :

I. Étude de la descendance. - Cette méthode est excellente. Encore, rencontrons-nous des difficultés du fait que, chez nos grands 
animaux, êtres complexes, nous connaissons imparfaitement les facteurs héréditaires qui sont à la base des caractères économiques les plus intéressants, du fait aussi que les animaux trop jeunes n'ont pas encore de descendants suffisamment développés ou à rendement contrôlable.

II. Contrôle du rendement. - Il faut signaler ici la variabilité du rendement dans les conditions ordinaires de la pratique, alors que les sujets observés ne se trouvent pas soumis aux mêmes influences de milieu, d'alimentation, de période de lactation, etc. Pour certains cas, il existe des coefficients de correction. Néanmoins, dans l'appréciation d'une descendance, il faut convenir que l'analyse des facteurs héréditaires est rendue bien difficile chez nos grandes espèces domestiques. Ajoutons que la qualité doit être contrôlée au même titre que la quantité, et qu'il faut souvent avoir recours pour le contrôle à des méthodes précises, utilisables seulement dans les laboratoires.

\section{Appréciation par les formes et signes extérieurs. -} Nous pouvons même faire appel, à titre complémentaire, au système des mensurations.

A la vérité, un examen détaillé, par cette dernière méthode, permettra bien de découvrir des types extrêmes, spécialisés pour la production du lait ou de la viande, pour la vitesse ou le travail lent et puissant, etc. Mais les données ainsi recueillies au sein d'une race sont encore insuffisantes et l'application des données biométriques a fait justice de maintes corrélations admises autrefois entre tels signes extérieurs et telle productivité (1).

IV. Étude de I'ascendance. - Celle-ci n'est possible que grâce aux nombreux renseignements puisés dans les livres généalogiques; elle fournit de fortes présomptions en faveur de la valeur d'un individu, mais ne donne pas de certitude.

$$
*^{*} *
$$

Rien d'étunnant done à ce que des expérimentateurs aient cherché à compléter nos possibilités d'examen de la "valeur d'élevage " et aient fait appel aux ressources apportées par d'autres sciences, telles que l'histologie, la physiologie, ete. L'étude du sang devait attirer immédiatement l'attention. C'est le tissu le plus important du corps : a priori, chaque sang doit être caractérisé par une série de coefficients biologiques individuels.

Avant de communiquer les résultats de nos recherches personnelles, nous ferons connaître les conclusions des plus récentes publi-

(1) Consulter notamment : "Contribution à l'étude de Ia valeur pratique des signes laitiers et beurriers ehez la vache n, par A.-MI. Lerox. (Réf. 6-b.). 
cations afin de permettre d'appréeier les méthodes modernes de recherches (1).

Du point de vue histologique, d'après von MaLsburG, une petite cellule possède plus de vitalité qu'une grande cellule. parce que sa surface est plus grande comparativement à sa masse totale, et que e'est par la surface que la cellule opère ses échanges nutritifs avec le milieu liquide ambiant.

Dans son travail sur les relations des propriétés du sang avec l'âge, l'état gravide, la production du Jait, la race, le docteur RIorTer conclut :

"Avec une chute du rendement en lait (période de lactation) et l'élévation de la richess e en graisse, on constate une augmentation. de globules rouges en même temps qu'une réduction de la dimension de ces derniers. La teneur en hémoglobine de $100 \mathrm{ccm}$. de sang est la plus basse quand la teneur en matières grasses est la plus haute. La richesse en hémoglobine de chaque globule subit une diminution parallèle à la chute du rendement en lait et à l'augmentation de la richesse. Un poids spécifique élevé du sang est en corrélation avec une faible richesse du lait.

“ Plus le lait est riche, plus élevé est le pourcentage des lymphocytes et monocytes et plus faible est le pourcentage des neutrophiles et eosinophiles et inversement. $)$

Du point de vue physiologique, résumons les conelusions du dernier travail de Kronacker et de ses collaborateurs. (Rét. 10 et 11).

Ces auteurs n'ont pas trouvé de relation entre les mensurations du corps et la richesse du lait, mais bien entre la quantité du lait et deux mensurations qui sont : la hauteur aux hanches et la profondeur de la poitrine. D'autre part, il n'y a pas de corrélation négative entre la quantité du lait produite et la largeur de poitrine, non plus qu'avec le périmètre du canon. La poitrine plate n'est donc pas un indice laitier. Ce fait est en parfait accord avec le travail de l'un de nous sur le rapport entre la surface de section du tronc en arrière des coudes et le rendement en lait.

Les propriétés du sang se tiennent dans une dépendance étroite. Une haute teneur en hémoglobine conditionne une haute teneur en matières sèches. Si le nombre de globules rouges monte, la viscosité du sang augmente également. et la vitesse de sédimentation des globules rouges diminue.

On constate des variations nettes de certaines propriétés du sang quand on les rapproche des mensurations. Plus la poitrine est large, plus la teneur en matières sèches est élevée. IJ en est de même pour le périmètre du canon.

(1) Pour la littérature, consulter les numéros de Kühn-Archiv et de Zeitschrift für Züchtung, de ces dernières années. 
Un coefficient de corrélation nettement élevé existe entre la réserve alcaline et le périmètre du canon. Il est quatre fois aussi élevé que l'erreur probable.

Toutes ces longues et patientes recherches dont nous ne donnons qu'un rapide aperçu n'ont pas résolu le problème de la constitution envisagée au point de vue physiologique. Leurs auteurs ne se sont pas trouvés en mesure d'en tirer des indications pratiques relatives à la détermination de la productivité.

Il faudra, disent-ils, chercher de nouvelles méthodes.

La grosse difficulté de ce genre de recherches consiste, selon nous, dans la mise au point de J'influence exercée par les facteurs divers téls que l'âge, la période de lactation, la race, l'état d'embonpoint, les circonstances dù prélèvement, etc. A côté des facteurs sanguins étudiés par les savants allemands, le champ encore inexploré de l'étude des lipoïdes nous laissait l'espoir de découvrir un moyen pratique de distinguer entre eux les divers sujets d'un élevage.

La lecture du magistral traité du professeur Ch. PoRCHER, sur le "Procès de la matière grasse du lait ", dans lequel figurent des vues prophétiques sur ce sujet, nous a incités à entreprendre l'examen de la variation des lipoïdes sanguins, chez les animaux de souche laitière, avec, comme objectif principal, la sélection beurrière des jeunes reproducteurs mâles.

Voici les premiers résultats obtenus :

TABLEAU I.

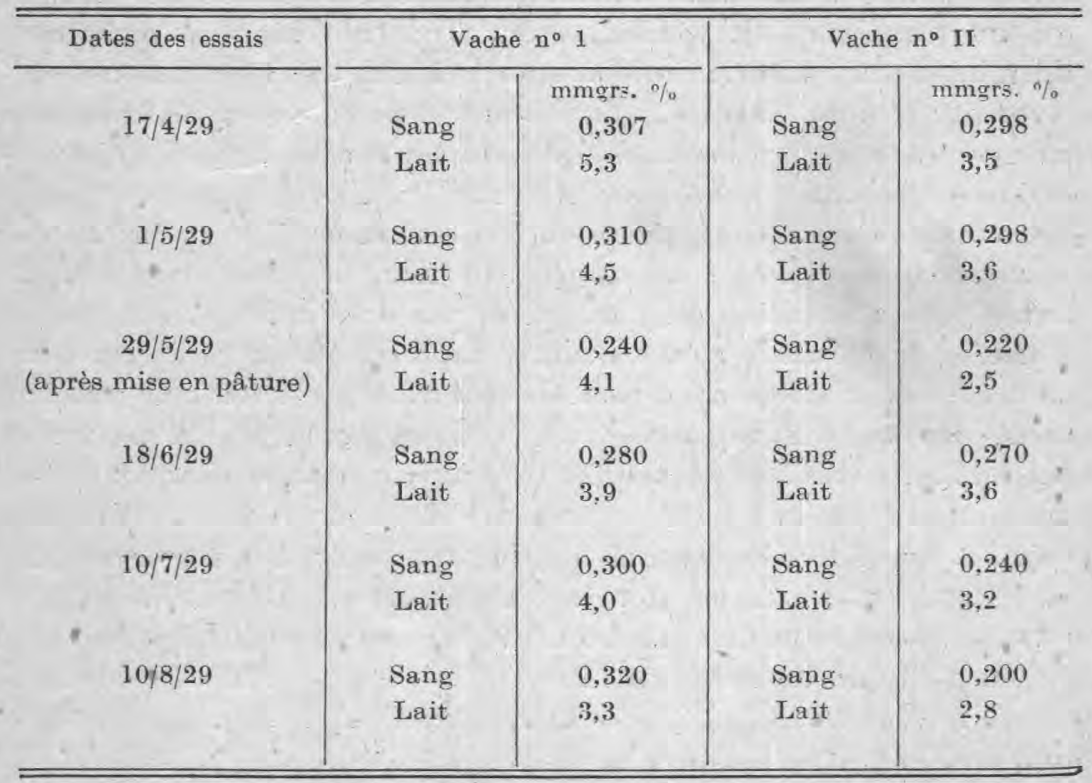


Des résultats encourageants furent notamment observés aussi sur deux taurillons issus de ces deux vaches. Nous pûmes constater que le taurillon à taux butyreux sanguin le plus élevé était fils de la vache No 1 .

Nous décidâmes alors d'envisager le problème avec toute la rigueur expérimentale désirable. Tout d'abord, et bien que des auteurs admettent qu'il y ait corrélation entre la teneur du sang en lipoïdes et en acides gras, il convenait de séparer ces derniers de l'insaponifiable.

D'autre part, il était préférable de faire porter nos recherches sur des races françaises bien distinctes, moins mélangées que les races belges, afin d'écarter autant que possible l'influence de la dominance, mendélienne ou non, de caractères sanguins, à l'instar de ce que l'on observe dans les phénomènes d'isoagglutination. En outre, nous avons pu profiter des services de la Station volante de recherches de l'Office régional du Nord, dont la mobilité et la souplesse d'action se prêtaient fort bien à ce genre de travaux.

\section{Le matériel animal}

Grâce à l'amabilité de trois éleveurs, MM. Grard, Mercier et Morei, nous avons eu la bonne fortune d'étudier des animaux de trois races différentes; M. J.-E. LuCAs nous a permis d'effectuer de nombreux prélèvements de sang sur deux sujets de son étable à Gournay-sur-Marne. Nous devons à M. le professeur VALĹ̂́E et à son adjoint, M. RINJARD, d'avoir pu poursuivre des expériences de quelque durée sur deux vaches bretonnes du Laboratoire de recherches du ministère de l'Agriculture, à Alfort. Enfin, des échantillons, prélevés par l'un de nous dans une ferme de Belgique, ont été analysés en même temps que les autres prélèvements au laboratoire de zootechnie de l'Institut National Agronomique.

L'étable de M. GIARD est peuplée de vaches jerseyaises. Placée dans une forêt, elle ne dispose que de mauvais pâturages, dont la faible valeur nutritive est compensée par des fourrages achetés au dehors. Les animaux sont contrôlés depuis 1926 par le Service du contrôle laitier de Seine-et-Oise. Le contrôle y est fait une fois par mois, pendant vingt-quatre heures, et porte non seulement sur la production laitière et le taux butyreux du lait de chaque vache, mais encore sur la valeur fourragère des rations individuelles. Les sujets examinés, mâles et femelles, appartenaient à la même famille, et avaient entre eux des liens de parenté étroits. Ils ont été saignés une fois, dans la matinée, le 26 octobre.

La vacherie de M. Mercier, de Quiévy, est remarquablement conduite. Les animaux y donnent des rendements laitiers élevés (5 à $6.000 \mathrm{~kg}$, en moyenne). Issus des familes les plus réputées de la 
race hollandaise frisonne, leur valeur beurrière est nettement supé rieure à la moyenne de cette race. Le contrôle est fait une fois par mois, par l'agent du syndicat local. L'année dernière, des essais entrepris par la Station volante d'expérimentation ont permis de recueillir des données à la fois précises et complètes sur le système d'alimentation adopté par le propriétaire. Lors des premiers prélèvements, Je 24 octobre, le troupeau allait encore en pâture, sur d'excellents herbages. Une deuxième série de saignées a été faite le 10 mars, vers la fin de la période de stabulation permanente.

M. Morel possède un important troupeau de bétail flamand, issu d'une souche sélectionnée depuis de longues années. Nous avons pu trouver chez lui un lot de sujets homogènes sous le rapport des formes corporelles et de l'aptitude beurrière, descendants d'un même taureau. Nous avons eu ainsi la chance de saigner au même moment sept vaches et un taureau issus d'un même père, ainsi que quatre mâles et une femelle nés de ces vaches et de leur propre frère. Pour toutes les vaches de ce troupeau, nous avons pu nous procurer les renseignements relatifs à leur rendement laitier et beurrier auprès du syndicat départemental de la Somme, dont la création remonte à l'année 1925. M. MorEL, qui a suivi nos essais avec un vif intérêt, nous a fourni des résultats très complets et très détaillés concernant l'alimentation de ses animaux. Une première série de prélèvements a été faite chez lui le 21 octobre. Lors de la seconde, effectuée le 11 mars, un certain nombre de sujets ont été saignés deux fois, à deux heures différentes de la journée.

A l'étable de M. J.-E. LuCAs et au laboratoire d'AJfort, des prises de sang, faites à des intervalles de trois à quatre heures, ont eu pour but de rechercher s'il existait une influence quelconque de l'heure du prélèvement sur le taux de matières grasses des échantillons de sang prélevés au cours de la même journée, chez la même vache.

\section{La méthode d'analyse}

Au cours d'essais préliminaires, tentés par l'un de nous sur les vaches du troupeau de l'Institut Agronomique de Gembloux, les analyses de sang avaient été faites par la méthode de GotTLIEB. A la réflexion, les critiques que n'aurait pas manqué de soulever l'emploi de cette méthode simpliste nous ont décidé à l'abandonner. Sur le conseil de M. LEMOIGNe, professeur de chimie analytique à l'Institut National Agronomique, nous avons adopté la méthode décrite par LEMELAND, (1) laquelle représente un perfectionnement de la méthode classique de Kumagawa et Suto. Voici la description succincte de notre mode opératoire.

(1) Voir Référ. 1, 2, 3. 
a) Prélèvement. - - L - sang est prélevé par le moyen d'une fine aiguille de $1 \mathrm{~mm}$. de diamètre ; il est reçu directemont dans une fiole jaugée de $25 \mathrm{~cm}^{3}$, dans laquelle on a placé une très petite pincée de citrate de sodium réduit en poudre fine. Après avoir enlevé l'excès de sang dans le col du flacon à l'aide d'une pipette pour amener l'affleurement du liquide au trait de jauge, l'échantillon est versé lentement dans un flacon à large ouverture de $250 \mathrm{~cm}^{3}$, bouchable à l'émeri, dans lequel on a placé $50 \mathrm{~cm}^{3}$ d'alcool à $95^{\circ}$. Les flacons convenablement étiquetés sont ramenés au laboratoire et conservés dans un endroit frais jusqu'au moment de leur dosage.

b) Extraction. - Le coagulum en suspension dans l'alcool est recueilli sur un filtre à plis. L'alcool de déshydratation est soigneusement mis à part.

Le filtre et son contenu sont alors placés dans la capsule d'un appareil de Kumagawa. L'extraction à l'alcool à $95^{\circ}$ est conduite pendant huit heures. Les liquides d'extraction et-l'alcool de déshydratation, mélangés ensemble, sont évaporés à sec au bain-marie en s'aidant du vide, afin d'éviter l'oxydation des acides gras non saturés.

c) Saponitication - Dana le ballon même qui a servi à l'évaporation, ajouter $25 \mathrm{~cm}^{3}$ d'une solution titrée de potasse deux fois normale, dans l'alcool à $95^{\circ}$. On chauffe ensuite pendant deux houres, en ramenant l'aleool dans le ballon à l'aide d'un réfrigérant de Vigreux. Verser alors dans le liquide $40 \mathrm{~cm}^{3}$ d'acide chlorhydrique normal, puis $26,2 \mathrm{~cm}^{5}$ d'aleool à $95^{\circ}$ et $8,8 \mathrm{~cm}^{3}$ d'eau. Après avoir chauffé de nonveau à reflux pendant vingt minutes, on obtient la séparation des matières insaponifiables.

La liquide est transvasé sans perte dans une ampoule à décantation de $500 \mathrm{~cm}^{3}$, avec le liquide de lavage, formé de potasse décinormale dans l'alcool à $50^{\circ}$.

A quatro reprises différentes, avec des quantités d'éther de pétrole de 100 , 50,40 et $40 \mathrm{~cm}^{3}$, on procède ensuite à l'extraction de l'insaponifiable (point d'ébullition de l'éther' : $60^{\circ}$ ). L'éther est ensuite lavé avec $50 \mathrm{~cm}^{2}$ d'eau distillée, dans une ampoule à décantation de $250 \mathrm{~cm}^{3}$. Après décantation, l'eau est ajoutée au liquide hydroalcoolique, qui renfermn les substances grasses sous forme de savons.

d) Dosage de l'insaponitiable total (cholestérine). - L'extrait éthéré est évaporé à siceité dans un ballın, de préférencesn s'aidant du vide. Le résidu est dissout dans $10 \mathrm{~cm}^{3}$ d'alcool absolu, renfermant $\mathrm{l} \mathrm{cm}^{3}$ de soude alcoolique décinormale, puis transvasé sans perte dans un cristallisoir à bec de $60 \mathrm{~mm}$. de diamètre, dont on se sert pour les lavages d'alcool absolu.

Evaporer de nouveau au bain-maris, sécher une heure à l'étuve réglée à $50^{\circ}$. Couvrir le résidu sec d'éthær de pétrole, filtrer le liquide sur amiante. Peser l'extrait sec final après séchage à $50^{\circ}$ pendant une heure. On obtient ainsi le poids de cholestérine correspondant ì $25 \mathrm{~cm}^{3}$ de sang. Le résultat de la pesée est d'environ 60 milligrammes.

e) Dosage des acides gras, - Lo liquide hydroalcorlique est recueilli dans un bécher de $400 \mathrm{em}^{3}$, puis évaporé au kain-marie, jusqu'à la réduction à la moitié de son volume initial. Transvaser ensuite dans une ampoule à décantation, après refroidissement.

A sidifier nottement par l'acide chlorhydrique au tiers. Faire trois extractions avec de l'éthər sulfuriqus en employant suecessivement, dans l'ordre indiqué, las quantités d'éther ci-après : $100 \mathrm{~cm}^{3}, 50 \mathrm{~cm}^{3}, 50 \mathrm{~cm}^{3}$. 
Les extraits éthérés, réunis dans un même ballon et additionnés de $10 \mathrm{~cm}$ d'alcool absolu, sont évaporés dans le vide. L'alcool est éliminé au bain-marie et le résidu est rapidement séché par immersion rapide du ballon dans l'eau bouillante.

On reprend par 30 à $40 \mathrm{~cm}^{3}$ d'éther de pétrole, versés dans le liquide encore chaud. Laisser reposer quelques heures pour que les impuretés se déposent. Filtrer au creuset de Gooch, laisser l'éther s'évaporer à l'air libre, puis sécher dans une étuve soigneusement réglée à $50^{\circ}$. Peser après un court séjour dans l'exsiccateur à l'acide sulfurique. Le poids d'acide gras recueilli, en partant d'une prise de sang de $25 \mathrm{~cm}^{3}$, est d'environ 60 milligrammes.

En dépit de sa complexité, la méthode ci-dessus décrite se prête assez bien aux dosages en série, à la condition de posséder un nombreux matériel et de travailler avec ordre. Nous n'entreprendrons pas ici la discussion des avantages de cette technique, comparée à celles de Kumagawa-Suto et WindHaus, modifiées par Mayer et SchaEFfER, et employées par E. TERRorne dans son magistral travail intitulé "Contribution à la connaissance de la physiologie des substances grasses e lipoïdiques » (1). D'après Lemeland, les perfectionnements apportés à l'ancienne technique permettraient d'éviter l'oxydation des acides gras et de prévenir l'entraînement des impuretés. Nous devons ajouter que presque tous nos dosages ont été faits en double et que les résultats de chaque couple d'épreuves n'ont montré qu'exceptionnellement des écarts supérieurs à $1 \mathrm{mmgr}$.

\section{Nombre et nature des observations recueillies}

$\mathrm{Au}$ total, nous avons recueilli et analysé, sur 44 bêtes différentes, 87 échantillons de sang, presque tows en double exemplaire. Pour chacun des animaux étudiés, des fiches reportées à la fin de notre travail font connaître le sexe, l'âge, le pédigree, les rendements laitiers et beurriers calculés d'après le contrôle, les dates et heures des pr lèvements de sang, ainsi que les résultats d'analyse de chaque échantillon. Lorsque plusieurs prélèvements de sang ont été faits sur le même animal, nous avons procédé au calcul des teneurs moyennes en acides gras, chlorestérine et lipoïdes totaux correspondant au sang du sujet considéré.

\section{Variations de l'indice lipémique et du coefficient lipémique chez les bovidés}

D'après E. Terroine, la quantité de lipoïdes présente dans le sang d'un même animal tend à demeurer constante. Cette quantité constitue l'indice lipémique. D'autre part, le rapport de la cholestérine aux acides gras, qui présente de grandes différences chez les divers sujets d'une même espèce, tend à demeurer sensiblement constant

(1) Voir rja. 4. 
chez le même individu. Terroine donne à ce rapport le nom de coefficient lipémique.

Dans quelle mesure ces résultats peuvent-ils s'appliquer aux bovidés ? Les données numériques nombreuses que nous avons recueillies, sur des animaux qui ont été saignés à plusieurs reprises, dans des conditions variables de lactation, d'âge et d'alimentation, fournissent une réponse à cette question. Le tableau ei-après donne les valeurs extrêmes des indices lipémiques et coefficients lipémiques constatés chez les mêmes individus.

TABLEAU II.

VARIATIONS DE L'INDICE LIPÉMIQUE ET DU COEFFICIENT LIPÉMIQUE CHEZ LES BOVIDÉS.

\begin{tabular}{|c|c|c|c|c|c|c|c|}
\hline \multirow{2}{*}{$\begin{array}{c}\text { Désignation } \\
\text { des } \\
\text { animaux } \\
\text { Sexe }\end{array}$} & \multirow[b]{2}{*}{$\begin{array}{l}\text { Nu- } \\
\text { méro }\end{array}$} & \multicolumn{3}{|c|}{ Indices lipémiques } & \multicolumn{3}{|c|}{ Cœlficients lipémiques } \\
\hline & & $\begin{array}{l}\text { Le plus } \\
\text { faible }\end{array}$ & $\begin{array}{l}\text { Le plus } \\
\text { fort }\end{array}$ & $\begin{array}{l}\text { Rapport du } \\
\text { plus fort au } \\
\text { plus faible } \\
\times \text { par } 100\end{array}$ & $\begin{array}{l}\text { Le plus } \\
\text { faible }\end{array}$ & $\begin{array}{c}\text { Le plus } \\
\text { fort }\end{array}$ & $\begin{array}{l}\text { Rapport du } \\
\text { plus fort au } \\
\text { plus faible } \\
\times \text { par } 100\end{array}$ \\
\hline \multirow[t]{21}{*}{ Fem. } & 1 & 3.76 & 4.06 & 108.0 & 0.69 & 1.16 & 168.0 \\
\hline & 2 & 5.64 & 6.78 & 120.2 & 1.10 & 1.59 & 144.4 \\
\hline & 3 & 5.28 & 6.22 & 117.8 & 1.21 & 1.83 & 151.2 \\
\hline & 4 & 3.16 & 4.28 & 135.4 & 1.02 & 1,52 & 149.1 \\
\hline & 5 & 4.60 & 6.08 & 132.1 & 0.60 & 0.86 & 143.3 \\
\hline & 6 & 5.51 & 5.64 & 102.3 & 0.91 & 1.04 & 114.2 \\
\hline & 7 & 5.22 & 5.59 & 107.1 & 1.04 & 1.14 & 109.7 \\
\hline & 8 & 4.98 & 5.48 & 110.0 & 1.05 & 1.08 & 102.8 \\
\hline & 9 & 4.29 & 4.40 & 102.6 & 0.83 & 1.01 & 121.6 \\
\hline & 10 & 4.08 & 4.36 & 106.8 & 0.69 & 1.12 & 162.5 \\
\hline & 11 & 4.28 & 5.07 & 118.4 & 0.82 & 1.04 & 126.8 \\
\hline & 12 & 4.02 & 4.64 & 115.4 & 1.11 & 1.19 & 107.3 \\
\hline & 13 & 4.12 & 4.96 & 120.4 & 0.78 & 1.48 & 189.5 \\
\hline & 14 & 4.62 & 4.78 & 103.4 & 1.17 & 1.43 & 122.2 \\
\hline & 15 & 4.98 & 5.26 & 105.6 & 1.02 & 1.03 & 101.0 \\
\hline & 16 & 4.02 & 4.44 & 110.5 & 0.95 & 1.07 & 111.3 \\
\hline & 17 & 4.70 & 5.10 & 108.5 & 0.82 & 0.89 & 108.5 \\
\hline & 18 & 4.90 & 5.06 & 103.2 & 0.89 & 1.04 & 116.9 \\
\hline & 19 & 4.38 & 5.02 & 114.6 & 1.21 & 1.41 & 116.5 \\
\hline & 20 & 4.58 & 4.64 & 101.3 & 1.29 & 1.39 & 107.9 \\
\hline & 21 & 4.20 & 4.40 & 104.8 & 1.06 & 1.20 & 113.2 \\
\hline \multirow[t]{6}{*}{ Mâle } & 2 & 3.72 & 4.62 & 124.2 & 0.79 & 1.89 & 176.2 \\
\hline & 3 & 3.40 & 4.46 & 131.2 & 0.70 & 1.14 & 162.8 \\
\hline & 4 & 3.94 & 4.16 & 105.6 & 0.91 & 1.60 & 175.8 \\
\hline & 6 & 4.02 & 4.82 & 119.9 & 0.91 & 1.30 & 142.9 \\
\hline & 9 & 6.04 & 6.62 & 109.6 & 1.04 & 1.09 & 104.9 \\
\hline & 10 & 4.57 & 5.28 & 115.5 & 1.06 & 1.44 & 135.9 \\
\hline
\end{tabular}


L'examen de ce tableau confirme la sensible constance de l'indice lipémique. Tandis quel'écart entre les taux de lipoïdes d'un même sujet, mesuré par le rapport du nombre plus élevé au nombre le plus faible, ne dépasse pas 136 (chiffre qui paraît d'ailleurs exceptionnel), l'écart correspondant entre le plus élevé $(6,02)$ et le moins élevé $(3,16)$ des indices de la première colonne de gauche du tableau (indices les plus faibles) est de 191. Il existe donc des individus à lipoïdes sanguins élevés (voir notamment les femelles nos 2 et 3 ainsi que le mâle no 9 ), qui peuvent se rencontrer dans la même étable avec des sujets beaucoup moins favorisés sous ce rapport (vaches nos $4,9,10,12$; taureaux nos 3 et 4 ).

Le coefficient lipémique présente des variations beaucoup plus considérables. L'assez grande irrégularité constatée dans les chiffres relatifs à la cholestérine explique en partie ces écarts. Certes, le tableau I nous montre que chez plus de la moitié des bêtes examinées (14 sur 26 ), il est possible de constater une certaine constance du rapport $\frac{\text { cholestérine }}{\text { acides gras }}$ mais les autres, et notamment les sujets F 10, F 13 et M 2, M 3, M 4, fournissent des chiffres à première vue déconcertants. Aussi, pour mettre en évidence l'existence d'un rapport physiologique entre les quantités de cholestérine et d'acides gras présentes dans le sang au même moment, nous avons imaginé de calculer, entre tous les couples de données recueillies, au nombre de 87 , le coefficient de corrélation correspondant. Voici les éléments de ce calcul ; mais auparavant, nous tenons à donner quelques renseignements sur la méthode des corrélations.

(A suivre).

\section{LA PASTEURISATION TUBULAIRE INTÉGRALE :}

GELLE QUI PREND LE LAIT DANS LE BAG DE RÉGEPTION, LE PASTEURISE ÉCONOMIQUEMENT, LE REFROIDIT ET LE GONDUIT A L'ABRI DE L'AIR, SANS PERTE PAR ÉVAPORATION, EN LUI CONSERVANT LES GAZ DISSOUS, DANS LES BIDONS DE TRANSPORT OU DANS LES BACS DE GARDE.

\section{par}

\section{H. CORBLIN,}

Ingénieur agronome-Constructeur

En janvier 1929, j'ai décrit dans Le Lait un procédé qui permet d'obtenir très simplement le refroidissement et même la congélation du lait sitôt la traite, afin de pouvoir le conserver et le consommer cru ou bien employer ce lait dans d'excellentes conditions pour une fabrication quelconque. Ce procédé réduit les chances de contamination de même que la main-d'œuvre autant que faire se peut, puisqu'il ne demande qu'un nettoyage soigné des seaux de traite et des bidons de transport. 